disease, however considerable inequalities remained. Other possible explanations for this vulnerability to disability among Indian Asian people will be discussed. Such inequalities are likely to have a detrimental impact on quality of life and morbidity in later years, and therefore, more research is urgently needed to understand these large ethnic inequalities in disability.

\section{OP32 HEALTH INEQUALITIES IN JAPAN 1986 TO 2007 BASED ON SELF-RATED HEALTH, HOUSEHOLD INCOME AND A NOVEL OCCUPATIONAL CLASSIFICATION}

doi:10.1136/jech-2012-201753.032

${ }^{1} \mathrm{~A}$ Hiyoshi, ${ }^{1} \mathrm{MJ}$ Shipley, ${ }^{2} Y$ Fukuda, 'EJ Brunner. ${ }^{1} E$ pidemiology and Public Health, UCL, London, UK; ${ }^{2}$ Community Health and Medicine, Yamaguchi University school of Medicine, Yamaguchi, Japan

Background While widening or stable inequalities have often been found in relation to economic stagnation in the west, time trends in health inequalities in Japan over the past two decades of economic stagnation are unclear. Comprehensive examination of temporal trends and factors contributing health inequalities in Japan are imperative to population health.

Methods We analysed a series of eight nationally-representative samples (Comprehensive Survey of the Living Conditions of People on Health and Welfare) between 1986 and 2007 (n=398,303). We used household income and a novel theory-driven occupational social class classification to calculate temporal trends in relative and slope indices of inequality [RII and SII, respectively] in self-rated fair or poor health. Multiple imputation was conducted to account for missing income data. Effects of living conditions, behavioural and psychosocial factors were tested using 1998 and 2001 samples. Results Overall, age-standardised self-rated fair or poor health showed U-shaped time trends in both sexes (quadratic term: men $p<0.001$, women $p=0.005$ ), with the lowest prevalence in early/mid 1990s. In men, income RII and SII narrowed significantly over the period (RII declined $1.4 \%$ per year, $\mathrm{p}=0.001$; SII declined $0.1 \%$ per year, $\mathrm{p}=0.031$ ). Inequalities in women's income were stable (RII and SII). The two indices showed constant inequalities across occupational social class in both sexes. After multiple imputation, point estimates became smaller, and narrowing of income inequality over time was significant for RII and SII in both sexes $(p<0.05, n=490,632)$. The annual declines were $1.4 \%$ and $1.0 \%$ for RII in men and women, respectively, and $0.1 \%$ for SII in both sexes. Living conditions, behavioural and psychosocial factors accounted for $28 \%$ of men's and $51 \%$ of women's health inequalities in Japan.

Conclusion Health inequalities in Japan either narrowed or remained stable from 1986 to 2007, in contrast to studies in western countries. The evidence provided by these analyses suggests that the prolonged economic stagnation in Japan had adverse effects at both ends of socioeconomic hierarchy. Potential explanations include effects of decreased income and increased stress in relation to job insecurity and workload across socioeconomic groups.

\section{Public Health Interventions: Transport}

\section{OP33 EXPOSURE-BASED ASSESSMENT OF MODAL TRAVEL RISK IN ENGLAND USING ROUTINE HEALTH DATA}

doi:10.1136/jech-2012-201753.033

${ }^{1} \mathrm{JS}$ Mindell, ${ }^{2} \mathrm{D}$ Leslie, ${ }^{3} \mathrm{M}$ Wardlaw. ${ }^{1}$ Epidemiology \& Public Health, UCL, London, UK; ${ }^{2}$ Centre for Physical Activity and Nutrition Research, Deakin University, Burwood, Australia, ${ }^{3}$ Edinburgh, UK

Background Cycling can make large contributions to population health but perceived road danger is a strong disincentive to cycling. Research on cycling safety is distorted by not making like-for-like comparisons. We examined age- and sex-specific deaths and injuries in England by travel mode, considering both the ICD external codes included in official travel risk data and those excluded but required for accurate comparative assessment, to provide more precise comparisons of travel risks by mode.

Methods ICD-10 external codes were grouped by type of incident for pedestrians, cyclists, and car/van drivers; the fourth digit was used to exclude non-transport casualties. Numbers of hospital admissions, from Hospital Episodes Statistics data, and deaths in England 2007-2009 were obtained for these ICD codes, by agegroup and sex. Aggregated per capita distance travelled by agegroup, sex and mode in England from National Travel Survey for each year 2007-2009, weighted to be nationally-representative, was multiplied by the estimated mid-year population. Time spent travelling was estimated using mean trip speeds by mode. We calculated fatal injury and hospital admission rates by distance travelled and by time spent travelling by age-group, sex, and travel mode. $95 \%$ confidence intervals for Poisson parameters were calculated using the formulae for weighted sums.

Results Fatalities per million hours' use ( $\mathrm{f} / \mathrm{mhu}$ ) varied little (0.15-0.45f/mhu by mode for men, $0.09-0.31 \mathrm{f} / \mathrm{mhu}$ for women). Risks were similar for men aged 21-49 years for all three modes and for female pedestrians and drivers aged 21-69 years. The group most at risk for each mode were: male drivers aged 17-20 years $(1.3 \mathrm{f} / \mathrm{mhu}, 95 \%$ CI 1.2, 1.4); male cyclists aged 70 years or older $(2.2 \mathrm{f} / \mathrm{mhu}, 1.6,3.0)$ and female pedestrians aged 70 years or older $(0.95 \mathrm{f} / \mathrm{mhu}, 0.86,1.1)$. In general, fatality rates were substantially higher amongst males than females, except for drivers aged 60 years or older. Risks per hour for male drivers under 30 years were similar or higher than for male cyclists; for 17-20 year olds the risk was higher for drivers $(33 / \mathrm{Bn} \mathrm{km}, 95 \%$ CI 30,$36 ; 1.3 \mathrm{f} / \mathrm{mhu}, 1.2$, 1.4) than cyclists $(20 / \mathrm{Bn} \mathrm{km}, 10,37 ; 0.24 \mathrm{f} / \mathrm{mhu}, 0.12,0.45)$ using distance or time.

Conclusion This is the first study in the UK to provide travel casualty rates by distance travelled and per hour, by mode, by age-group and sex, based on nationally-representative data, to enable unbiased intermodal comparisons for population sub-groups. Males aged 17-20 years old face higher risks as drivers than as cyclists, and do not achieve better safety as drivers until over 30 years. Not making like-for-like comparisons sustains the misleading stereotype that cycling is relatively hazardous.

\section{OP34 INEQUITIES IN BICYCLE USAGE: SOCIO-DEMOGRAPHIC PREDICTORS OF UPTAKE AND USAGE OF A PUBLIC BICYCLE SHARING SCHEME IN LONDON, UK}

doi:10.1136/jech-2012-201753.034

F Ogilvie, A Goodman. Nutrition and Public Health Interventions Research Department, LSHTM, London, UK

Background Cycling confers individual and population-level health benefits. Increasing numbers of cities are therefore introducing public bicycle sharing schemes, with London's Barclays Cycle Hire (BCH) scheme introduced in July 2010. Yet uptake of cycling is not equitable across socio-demographic groups. We sought to examine the characteristics of the BCH scheme's registered users, and to explore characteristics associated with usage.

Methods For users registering in the first seven months of the scheme, we obtained complete $\mathrm{BCH}$ registration and usage data, comprising: title (from which we inferred gender); home postcode; date of registration; access type; and number of trips made. We used postcodes to calculate distance to the nearest 'docking station' and to assign small-area-level data on ethnic composition, income deprivation, and the prevalence of commuter cycling. We compared characteristics of registered users with local residents and workers in the inner-London area served by the scheme. We used multi-level linear regression to examine correlates of 'mean number of trips', and logistic regression to examine correlates of 'ever use'. 